\title{
Balkanologie
}

Balkanologie Revue d'études pluridisciplinaires

Vol. IX, n $1-2 \mid 2005$

Volume IX Numéro 1-2

\section{Transmission de l'identité et culte du héros}

Les associations de Turcs de Thrace occidentale en Allemagne

Handing down identity and hero cult : the Western Thracian Turks' associations in Germany

Jeanne Hersan

\section{(2) OpenEdition \\ Journals}

Édition électronique

URL : http://journals.openedition.org/balkanologie/581

DOI : 10.4000/balkanologie.581

ISSN : 1965-0582

Éditeur

Association française d'études sur les Balkans (Afebalk)

Édition imprimée

Date de publication : 1 décembre 2005

ISSN : 1279-7952

Référence électronique

Jeanne Hersan, «Transmission de l'identité et culte du héros », Balkanologie [En ligne], Vol. IX, n 1-2 I 2005, mis en ligne le 13 janvier 2010, consulté le 17 décembre 2020. URL : http://

journals.openedition.org/balkanologie/581; DOI : https://doi.org/10.4000/balkanologie.581

(c) Tous droits réservés 


\title{
TRANSMISSION DE L'IDENTITÉ ET CULTE DU HÉROS : LES ASSOCIATIONS DE «TURCS 》 DE THRACE OCCIDENTALE EN ALLEMAGNE
}

\author{
Jeanne Hersant*
}

\section{INTRODUCTION}

L'étude présentée ici est le résultat d'un travail de terrain effectué en Allemagne et en Thrace occidentale dans le cadre d'un doctorat ${ }^{1}$ ayant pour objet l'émigration des membres de la "minorité musulmane" de cette région du nord de la Grèce. Plus précisément, il s'agit de retracer l'histoire de la mobilisation identitaire impulsée depuis l'Allemagne, visant à la reconnaissance de cette minorité comme turque par les institutions européennes.

Il nous faut tout d'abord clarifier le choix de la dénomination utilisée ici pour qualifier la population étudiée. Il s'agit de la minorité officiellement reconnue comme " musulmane " par le traité de Lausanne de $1923^{2}$, est généralement décrite comme étant composée de trois groupes, alternativement présentés comme "ethniques" ou "linguistiques" : Turcs (ou turcophones), Pomaques (qui parlent un dialecte proche du bulgare) et Tsiganes (également turcophones). Dans les faits, la majeure partie de cette population utilise le

\footnotetext{
* Doctorante en sociologie à l'Ecole des hautes études en sciences sociales (Centre d'histoire du domaine turc) / ATER à l'Institut d'Etudes Politiques de Bordeaux. Contact : jeanne.hersant@ehess.fr

${ }^{1}$ Cette recherche a commencé en 2001 dans le cadre d'un DEA. Hersant (Jeanne), La stratégie européenne des Turcs de Thrace occidentale. Etude de cas : trajectoires migratoires, stratégies identitaires et mobilisations transnationales à travers la vie associative des Turcs de Thrace grecque en Allemagne et en GrandeBretagne, Paris : EHESS (mémoire réalisé sous la direction de Hamit Bozarslan), 2001.

${ }^{2}$ Le traité de Lausanne fut signé le 24 juillet 1923, quelques mois avant l'échange obligatoire de population entre la Grèce et la Turquie. Les musulmans de Thrace occidentale, ainsi que la minorité grecque orthodoxe de Constantinople (Istanbul) et des îles Imbros (Gökçeada) et Ténédos (Bozcaada) furent exclus de cet échange. La clause du traité concernant ces minorités énonce, sur le mode de la réciprocité, des droits positifs pour leurs membres. Les musulmans bénéficient de la juridiction civile et religieuse des mufti, d'un système d'enseignement bilingue, et de la gestion de leurs écoles et lieux de culte. Cette clause leur garantit par ailleurs les mêmes droits que les citoyens grecs de religion orthodoxe.
} 
turc comme langue véhiculaire, quelle que soit sa langue maternelle; mais dans certaines zones rurales le pormaque reste la langue vernaculaire, au moins dans la sphère privée.

Cette uniformisation linguistique est liée notamment à la gestion de cette minorité menée par les États grec et turc, incarnée entre autres par un système scolaire qui lui est propre, où l'enseignement se fait en grec et en turc. La perspective adoptée ici est celle du nationalisme turc et de la "turcité", dont nous mettrons en avant la variation semantique hors du contexte de la Turquie. La population "musulmane" de Thrace occidentale baigne dans les référents culturels de la République de Turquie, notamment à travers l'école et les associations, mais aussi par le biais de la presse locale. De plus, le discours politique en vigueur au sein de cette minorité - en Grèce comme dans l'espace migratoire est focalisé sur la reconnaissance collective de l'identité turque du groupe. En turc, ses membres sont dénommés Batı Trakya Türkleri ou Batı Trakyallar. $\mathrm{Ce}$ dernier terme les différencie nettement des Grecs (Yunanllar, Rumlar), tout en restant plus neutre car se rapportant simplement à la localisation géographique ${ }^{3}$. Cette nuance n'ayant pas d'équivalent en français, nous avons retenu l'expression de "Turcs" de Thrace occidentale sans que cela ait une quelconque prétention normative ni englobante. Plus généralement, le terme "musulmans" sera utilisé pour évoquer la minorité dans son ensemble ou de façon indéfinie, et "turcs" lorsqu'il sera spécifiquement question du mouvement identitaire et/ou associatif, et des acteurs qui y sont impliqués.

Les enquêtes présentées ici retracent l'aspect intra-européen des flux migratoires en provenance de Thrace occidentale dans le contexte de l'après crise de Chypre, et dans le cadre des accords d'envoi de main d'œuvre conclu entre la Grèce et l'Allemagne. Cet aspect du fait minoritaire en Thrace occidentale n'a pas été étudié à ce jour4. Ces enquêtes permettent d'appréhender le réseau associatif d'Allemagne ${ }^{5}$, et de reconstituer la stratégie des migrants pour alerter les institutions européennes sur la condition de la minorité "turque" de Thrace occidentale. Il s'agit parallèlement de comprendre comment le groupe se perpétue grâce à la transmission de codes de conduite et d'interdits visant à encadrer les comportements, en public mais aussi dans la sphère privée. Les associations interviennent alors en tant qu'organisatrices d'événements visant au rassemblement des membres du groupe, au cours desquels ces règles sont ré-

\footnotetext{
${ }^{3} \mathrm{En}$ turc, le suffixe -li/lu indique le fait d'appartenir à un endroit (et -lar indique le pluriel).

4 Seul l'ouvrage de Michael Ackermann relate un entretien collectif réalisé avec des Turcs de Thrace occidentale vivant en Allemagne. Ackermann (Michael), Die türkische Minderheit in West-Thrakien, Geschichte und Gegenwart, Bad Schussenried : Gerhard Hess-Verlag, 2000.

5 Ces enquètes ont ètè menèes en février et mars 2001, puis en février 2003, dans les villes suivantes : Berlin, Bonn, Düsseldorf, Giessen, Gütersloh, Viersen (Grefrath), Witten, D'autres ont eu lieu, avec les mèmes interlocuteurs, sur leur lieu de villégiature en Grèce, à Komotini (Gümülcine), en août 2002.
} 
affirmées. Enfin, les associations interviennent dans la production d'un imaginaire collectif, entretenu sur le mode de la sacralité autour de la figure du leader disparu, Sadık Ahmet.

Les entretiens menés dans le cadre de cette enquête de terrain l'ont été aussi bien dans des associations que chez des particuliers, sous forme de conversations parfois anodines, du moins en apparence; cela nous a permis de comprendre les pratiques sociales et les codes qui structurent le groupe. Il s'agissait de "privilégier l'expérience des acteurs en reconstruisant autour d'elle le contexte (ou plutôt les contextes) qui lui donnent sens et forme " 6 .

On présentera dans un premier temps la stratégie de mobilisation développée à l'échelle européenne, dans les années 1980 et 1990, par les associations de Turcs de Thrace occidentale en Allemagne. Il sera aussi question du rapport à la Turquie de leurs membres et dirigeants. Celui-ci est complexe, et il faut l'aborder à travers plusieurs niveaux pour en appréhender l'épaisseur, et surtout ne pas conclure trop rapidement à l'allégeance automatique à l'État turc de la part des acteurs concernés.

Dans une seconde partie interviendra le travail de terrain dans et hors cadre associatif, présentant les mécanismes par lesquels se perpétue le groupe, à travers divers lieux et moments de sociabilité, notamment les aile gecesi (littéralement : " soirées familiales "), qui sont autant des marchés matrimoniaux que des lieux d'expression politique.

Enfin, dans une troisième partie seront analysés les mécanismes visant à unifier le groupe autour de quelques symboles forts, à sacraliser personnages et événements politiques, notamment la figure du " martyr "Sadık Ahmet (voir biographie en annexe). Il ne s'agit pas ici de pratiques religieuses islamiques en tant que telles : nous nous trouvons, de façon beaucoup plus universelle, dans le domaine de la production de héros, saints et martyrs? ${ }^{7}$. Nous verrons que la production identitaire et le registre politique adopté prennent appui sur les structures sociales décrites, notamment les solidarités familiales et la religiosité - mẽme lorsque celle-ci n'est que diffuse.

\footnotetext{
${ }^{6}$ Revel (Jacques), "Présentation ", in Revel (Jacques), èd., Jeux d'échelles. La micro-analyse à l'expérience, Paris : Gallimard, 1996, p. 13.

7 Centlivres (Pierre), Fabre (Daniel), Zonabend (Françoise), éds., La fabrique des héros, Paris : Editions de la Maison des sciences de l'homme, 1998 ; Centlivres (Pierre), èd., Saints, sainteté et martyre : la fabrication de l'exemplarité, Neuchâtel / Paris : Editions de l'Institut d'ethnologie / Editions de la Maison des sciences de l'homme, 2001 ; Mayeur-Jaouen (Catherine), éd., Saints et héros du Moyen-Orient contemporain, Paris : Maisonneuve \& Larose, 2002.
} 


\section{LA MOBILISATION POLITIDUE DES TURCS DE THRACE OCCIDENTALE EN ALLEMAGNE}

\section{Historique des flux migratoires en provenance de Thrace occidentale}

Après l'officialisation des accords d'envoi de main d'œuvre entre l'Allemagne et la Grèce, en 1960, les départs furent massifs : " de 42000 ressortissants grecs recensés en 1961, [ils étaient] 408000 à la fin de septembre 1973, puis 298 ooo en $1980 n^{8}$. Pourtant, les entretiens menés ont révélé que c'est seulement à partir de 1969 que les musulmans de Thrace occidentale ont obtenu l'opportunité de partir travailler en Allemagne9. Si quelques personnes ont pu partir avant cette date (parfois hors du cadre des accords), la majorité de nos interlocuteurs ont dû attendre deux, trois, voire quatre ans avant que leur demande d'émigration soit satisfaite. Par contre, ceux qui ont postulé après 1969 ont été envoyés en Allemagne dans les semaines suivant l'acte de candidature.

Une seconde vague migratoire a eu lieu à partir de 1988 , lorsque la Grèce est devenue membre à part entière de la Communauté européenne ${ }^{10}$. C'est pourquoi il est d'autant plus malaisé de déterminer le nombre de musulmans originaires de Thrace occidentale qui vivent en Allemagne. La Fédération des Turcs de Thrace occidentale en Europe (Avrupa Batı Trakya Türk Federasyonu), qui regroupe une trentaine d'associations en Allemagne et une à Londres, revendique entre 20 et 30000 "compatriotes", mais ce chiffre n'est pas vérifiable. Les statistiques allemandes aussi bien que grecques ne tiennent pas compte du critère religieux et ne permettent pas d'évaluer l'importance de la composante

\footnotetext{
${ }^{8}$ Kolodny (Emile), Samothrace sur Neckar : des migrants grecs dans l'agglomération de Stuttgart, Aix-enProvence : Institut de recherches méditerranéennes / Centre d'études de géographie méditerranéenne, 1982, p. 10.

9 Cette mesure nous a été confirmée par une fonctionnaire du ministère grec des Affaires étrangères. Le seul document qui en fasse mention explicitement est un article de Gerassimos Notaras sur les migrations vers l'Allemagne depuis le département de l'Evros (frontalier de la Turquie) : " It should be noted here that emigration in the province of Evros would be even greater were not the State attempting to check it through administrative measures ", (Notaras, Gerassimos, "Research in the Province of Evros ", Migration Series, 1 (1) , 1967, p. 64). Une telle entrave à l'émigration est également évoquée dans les travaux de Lois Labrianidis sur la zone sous surveillance militaire. Cette dernière, abolie en 1997, englobait principalement les villages pomaques de la région de Xanthi (İskeçe). Voir Labrianidis (Lois), " The Impact of the Greek Military Surveillance Zone on the Greek Side of the Bulgarian-Greek Borderlands ", Boundary and Security Bulletin, 7 (2), 1999.

${ }^{10}$ La Grèce a rejoint la Communauté européenne en 1981, mais la libre circulation entre la Grèce et les autres pays-membres n'est devenue effective qu'en 1987, avec l'entrée en vigueur de l'Acte unique européen). Voir Hersant (Jeanne), " La minorité musulmane en Thrace et l'intégration européenne de la Grèce ", Etudes turques et ottomanes. Documents de travail, (9-10), 2001 [dossier " Empire ottoman et Turquie moderne (XIX ${ }^{\text {ème }}$-XX ${ }^{\text {ème }}$ siècles) n].
} 
musulmane de la population grecque établie en Allemagne. En Grèce, cela tient d'une part au fait que les critères linguistiques et religieux n'apparaissent pas dans les recensements ${ }^{11}$; d'autre part à la discrimination susmentionnée concernant l'envoi de travailleurs. En Allemagne, les musulmans de Thrace occidentale sont tout simplement considérés comme des citoyens grecs. Dans leurs contacts avec l'administration allemande, en revanche, les "musulmans" de Thrace occidentale sont souvent pris pour des ressortissants turcs $^{12}$.

Sans pouvoir généraliser, étant donnée l'absence de statistiques, soulignons le fait que la plupart de nos interlocuteurs de la première vague migratoire - hormis le président de l'association berlinoise qui vient de Xanthi - sont originaires de Komotini (Gümülcine) et sa région. C'est parmi les migrants de la deuxième vague (celle de la fin des années 1980 et du début des années 1990) que l'on trouve des travailleurs originaires du département de Xanthi (İskeçe). Nombre d'entre eux viennent des villages situés dans l'ancienne zone militaire fermée ${ }^{13}$. Coupés du monde extérieur, les villages compris dans ce périmètre ont accusé un retard économique flagrant par rapport au reste de la région, qui était alors elle-mème la plus pauvre de l'Europe des Quinze ${ }^{14}$. Pour fuir cet isolement, la migration des villages vers la ville éponyme de Xanthi (Iskeçe) a été significative à partir des années 1970. Toutefois, pour autant que nous ayons pu le constater, les habitants de ces villages n'ont pas postulé au départ vers l'Allemagne dans le cadre des accords de main d'ouvre.

Mentionnons enfin un autre type de migration, qui s'est développé grâce au principe européen de libre circulation des personnes, et qui concerne principalement les villages pomaques du département de Xanthi. Des filières migratoires spécialisées ont vu le jour, selon les villages, certaines vers les chantiers navals d'Allemagne et d'Europe du Nord, d'autre vers les serres des

\footnotetext{
${ }^{11}$ Labbé (Morgane), "Les nationalités dans les Balkans : de l'usage des recensements ", L'espace géographique, (1) 1997, pp. 35-48.

${ }^{12}$ Ainsi, le Bundesausländerbeauftragte (Délégué fédéral pour les étrangers) et ses antennes dans les différents Länder ont affirmé ne pas connaïtre cette population ni ses associations ; il en a ètè de mêrne avec les Ausländerbeiräte (conseils municipaux d'étrangers), notamment ceux de Bonn et Witten, alors méme que les conseils de ces deux villes comptent ou ont comptè des membres de ce groupe dans leurs rangs. A Witten se trouve en outre le siège de la Fédération des Turcs de Thrace occidentale en Europe

${ }^{13}$ Les villages pomaques de la région de Xanthi (ìskeçe) sont restés jusqu'en 1996 prisonniers de la "zone interdite", zone militaire instaurée par Metaxas en 1936 pour contrer le danger communiste dans toute la Grèce du Nord. Lorsque cette zone fut supprimèe, sous la pression des instances européennes, elle s'étendait sur la région montagneuse au nord des départements de Xanthi et du Rhodope. Labrianddis (Lois), art.cit.
}

${ }^{14}$ Hersant (Jeanne), art.cit. 
Pays-Bas 15 . Pour ce qui est de l'Allemagne, ces filières ont été développées par des entrepreneurs eux-mêmes originaires de ces villages, établis en Allemagne et employant des intermédiaires chargés du recrutement en Thrace. Il s'agit pour eux de bénéficier des opportunités économiques offertes par l'espace européen, en pratiquant le dumping social. Les travailleurs partent pour des périodes de trois à six mois et reviennent l'été au moment de la récolte du tabac, activité qui, le reste de l'année, échoit aux femmes. Ce type de migration saisonnière atteint des proportions telles qu'il a clairement des effets sur l'organisation économique et sociale des villages concernés. C'est pourquoi il constitue à notre avis un objet d'étude en soi et ne sera pas traité dans cet article, qui s'intéresse à la formation d'une diaspora en Allemagne, et à la mobilisation par le canal associatif ${ }^{16}$.

\section{Genèse du réseau associatif en Allemagne}

Le mouvement de création des associations de Turcs de Thrace occidentale en Allemagne doit être mis en rapport avec les deux vagues migratoires évoquées, s'inscrivant elles-mêmes dans des contextes différents. $\mathrm{Si}$, au cours des deux périodes distinguées, les structures associatives étudiées présentent à la fois les traits d'associations culturelles et d'organisations politiques, leurs activités tendent plus, selon l'époque, vers l'une ou l'autre de ces dimensions.

La toute première association a été fondée à Berlin en 1974, peu après la crise de Chypre, par un militant du Parti communiste grec (KKE). L'association n'a vécu que six mois en raison des divergences politiques de ses membres : le président refusait les approches répétées du consulat turc et surtout des " Loups Gris ", ultra-nationalistes turcs liés au Parti d'action nationaliste (Milliyetçi Hareket Partisi-MHP) ${ }^{17}$; or, la plupart des membres de l'association avaient de la sympathie pour ce mouvement. Désavoué et traité de " sale communiste ", le président a mis la clé sous la porte ${ }^{18}$.

\footnotetext{
${ }^{15}$ Cette filière, qui concernerait le village de Hebil, est mentionnée dans “ Meriç'in öte yakası : Batı Trakya Türkleri " (L'autre rive de l'Evros : les Turcs de Thrace occidentale), Atlas Dergisi, (129), décembre 2003. Nous avons dans le cadre de cette enquête rencontré principalement des gens du village de Kendavros (Ketenlik) ou Ehinos (Șahin), qui travaillent dans les chantiers navals en Allemagne, et parfois en France. ${ }^{16}$ À propos de la notion de diaspora appliquée au cas des Turcs de Thrace occidentale, voir Hersant (Jeanne), " L'élaboration d'un discours identitaire dans l'espace migratoire des Turcs de Thrace occidentale ", CEMOTI, (34), 2002.

${ }^{17}$ Au sujet de l'activisme des "Loups Gris " en Allemagne, voir Aslan (Fikret), Bozay (Kemal), Graue Wölfen heulen wieder. Türkische Faschisten und ihre Vernetzung in der BRD, Münster : Unrast-Verlag, 2000.

${ }^{18}$ Entretiens réalisés à Berlin, mars 2001 et mars 2003.
} 
Officiellement, c'est-à-dire du point de vue de la Fédération des Turcs de Thrace occidentale en Europe ${ }^{19}$, la première association a vu le jour en 1978 à Giessen, puis un petit noyau s'est développé autour des associations de Giessen, Düsseldorf, Homburg, Kelsterbach, Munich et Stuttgart ; ces associations ont un temps publié un journal du nom de Yeni Adım (Nouveau pas ${ }^{20}$ ). Elles ont envoyé, au cours des années 1980, des délégations plaider la cause des Turcs de Thrace auprès des institutions européennes. Parmi leurs membres fondateurs, un seul maîtrisait l'allemand et l'anglais, il est devenu l'interlocuteur des députés allemands et européens, ainsi que des membres de l'Assemblée parlementaire du Conseil de l'Europe. C'est auprès de ces associations que Sadık Ahmet a débuté ses activités militantes, en 1986. Lorsqu'une septième association, celle de Hambourg, a été créée, la Fédération a pu voir le jour en 1988, conformément aux dispositions juridiques allemandes. Nous avons recensé, en 2001, 31 associations de Turcs de Thrace occidentale en Allemagne $^{21}$. Au moins 25 associations ont donc été créées depuis 1988 , reflétant la croissance exponentielle du nombre d'arrivants jusqu'au milieu des années 1990.

\section{Mobilisation européenne et rapport à la Turquie}

Depuis la fin des années 1990, la mobilisation européenne des Turcs de Thrace a évolué. Les manifestations de rue et délégations envoyées au Parlement européen et au Conseil de l'Europe ont peu à peu fait place à une stratégie de lobbying, revendiquée et théorisée comme telle. Parallèlement à cela, en lien avec l'assouplissement du climat politique en Thrace, la mobilisation s'est déplacée des associations d'Allemagne vers celles de la Thrace. En

\footnotetext{
19 Notamment dans les " mémoires " de son premier président, aujourd'hui domicilié à Bursa ; il s'agit en fait d'une compilation de documents retraçant les temps forts de la mobilisation des acteurs associatifs vis-à-vis des institutions européennes. Voir Alioğlu (Cafer), Batı Trakya Davası́nın Avrupa Cephesi (19821994) (La dimension européenne de la lutte des Turcs de Thrace occidentale, 1982-1994), İzmir : Dokuz Eylül Üniversitesi, 1998.

${ }^{20}$ Reprenant ainsi le nom du journal fondé en 1926 par cinq lettrés dont Mehmet Hilmi, figure historique des Turcs de Thrace occidentale, qui fut également le fondateur de l'Union turque de Xanthi. Ce journal parut en caractère arabes jusqu'en 1936 (ou 1946 selon les sources) et est considéré comme le premier journal local digne de ce nom. Voir Saglam (Feyyaz), Batı Trakya Türkleri Basm-Yaym Tarihi (Histoire de la presse et de l'édition des Turcs de Thrace occidentale), München / İzmir : Bavyera Eyaleti Batı Trakya Türkleri Aile Birliği / Batı Trakya Araștırma Merkezi Yayınlan, 2002.

${ }^{21}$ Ce chiffre est sensiblement le même aujourd'hui. L'un de mes interlocuteurs a en outre évoqué une " association de Thrace occidentale " à Bonn, qui rassemblerait des Tsiganes et refuserait d'avoir des contacts avec l'association locale des Turcs (entretien avec le président du club sportif des Turcs de Thrace occidentale, réalisé à Bonn, mars 2001).
} 
effet, les associations " turques" - quoique non reconnues officiellement ${ }^{22}$ jouissent d'une plus grande liberté d'expression. On assiste à une professionnalisation des acteurs associatifs, formés dans les universités turques et britanniques, maitrisant l'anglais et acquis au répertoire d'action européen. Ces acteurs, notamment ceux de l'Association des diplômés d'université (Yüksek Tahsilliler Derneği), sont représentés dans tous les forums et programmes européens dédiés à la promotion des droits des minorités nationales, qui regroupent des ONG et " organisations de la société civile ", selon l'expression consacrée en turc.

Ce n'est pas la seule évolution qu'ait connu la mobilisation européenne des Turcs de Thrace occidentale : avec la création d'une fédération en 1988, les associations d'Allemagne se sont de facto retrouvées sous tutelle turque. Au cours de la première série d'entretiens réalisés en Allemagne, en février et mars 2001, nos interlocuteurs avaient affirmé ne vouloir avoir de liens ni avec les autorités turques ni avec les associations de Turcs de Thrace en Turquie, arguant du fait que les membres de ces associations sont désormais des citoyens turcs (ce qui n'est pas entièrement conforme à la réalité). Ils disaient vouloir agir exclusivement dans les cadres juridiques grec et européen. Or, au fil de nos recherches et des rencontres ultérieures avec ces mêmes personnes (à Komotini en août 2002 puis à nouveau en Allemagne en février 2003), des contradictions sont apparues dans les témoignages. Les liens entre les réseaux associatifs allemand et turc sont en fait étroits, comme le montrent les publications à usage interne, ou encore les différents " symposium internationaux " sur la Thrace occidentale, à prétention scientifique, organisés en Turquie au cours des années 1990 ; le dernier en date s'est tenu à Londres en 2000.

Il est peu à peu devenu évident que les allégations répétées de nos interlocuteurs quant à leur loyauté vis-à-vis de la Grèce, et leur prise de distance par rapport aux autorités turques, n'étaient que l'expression de ce qu'il est admis comme convenable de dire à l'observateur étranger. En effet, la légitimité du mouvement de protestation, et son succès auprès des instances européennes, dépendaient de l'assurance que la population concernée agissait bien dans le cadre européen du droit des minorités et non en rapport avec de supposées visées irrédentistes de la Turquie ${ }^{23}$.

\footnotetext{
${ }^{22}$ Les associations dont le nom comporte l'adjectif "turque" ont été interdites dans les années 1980. Elles ont toujours fonctionné de facto, proposant de nombreuses activités sportives et culturelles, comme nous avons pu le constater lors de nos séjours en Thrace, entre août 2002 et septembre 2004. Néanmoins, en janvier 2005, à l'issue d'un processus judiciaire ayant duré vingt ans, la Cour de cassation a confirmé l'interdiction de l'Union turque de Xanthi pour " raisons de sécurité nationale ", faisant ainsi jurisprudence pour les autres affaires en cours. "Batı Trakya'da "Türk" adına yasak getirildi " (En Thrace occidentale, interdiction portée au nom "turc"), Radikal, 14/01/05.
}

${ }^{23}$ Hersant (Jeanne), op.cit. 
Or la présence turque dans l'espace migratoire des Turcs de Thrace occidentale ne s'est pas manifestée seulement par la diffusion des théories de la " synthèse turco-islamique "24. L'orientation européenne de l'action protestataire des Turcs de Thrace occidentale semble avoir été en partie définie depuis la Turquie. À la fin des années 1970, les membres de l'association de Düsseldorf étaient menés par un militant maoïste qui avait entamé en Allemagne des études universitaires, prenant ainsi conscience du fonctionnement des institutions européennes ${ }^{25}$. Face au succès de la mobilisation, l'enjeu pour les autorités turques était d'en tirer parti tout en endiguant le prestige grandissant de son instigateur auprès des membres de la minoritè, en Allemagne mais aussi en Thrace. Rappelons que les années 1970 et 1980 en Turquie ont été marquées par une répression très dure à l'encontre des militants de gauche et d'extrême gauche. Le Parti communiste turc n'a été autorisé que dans les années 1990, après la chute de l'URSS ${ }^{26}$.

Ainsi le premier président de la Fédération, Cafer Alioğlu, aujourd'hui retraité à Bursa, est un ancien instituteur ayant présidé l'Union turque de Xanthi à la fin des années $195^{\circ}$. Ses propos laissent transparaître le fait qu'en raison de cette expérience passée il était connu des autorités turques comme des acteurs associatifs ; il aurait ainsi été coopté à la tête de la nouvelle structure ${ }^{28}$.

Pour autant, malgré le constat de cette collusion, les processus d'identification individuelle s'établissent entre plusieurs registres, et il faut distinguer la revendication de la turcité du rapport à la Turquie. Celle-ci constitue certes un substrat identitaire et culturel indéniable et Mustafa Kemal est, tout comme en Turquie, le référent par excellence, d'autant que, originaire de Thessalonique, il est présenté comme hemşehri (pays). Tout comme en Thrace, certaines associations organisent en Allemagne des activités pour célébrer les

24 Au sujet de la " synthèse turco-islamique ", voir Copeaux (Etienne). Espaces et temps de la nation turque. Analyse d'une historiographie nationaliste (1931-1993), Paris : Editions du CNRS, 1997, Pp. 75-102.

25 Cet homme vit aujourd'hui à Kornotini, nous l'avons rencontré à Istanbul en aout 2005.

${ }^{26}$ D'autres formations marxistes ont néanmoins vu le jour à la fin des années 1960, le Parti ouvrier de Turquie, aujourd'hui disparu, et la Confédération révolutionnaire des syndicats ouvriers (DisK), toujours en activité. Kazancıgil (Ali), "L'État, figure centrale de la modernité turque ", in Vaner (Semih), éd., La Turquie, Paris : Fayard / CERI, 2006, p. 141.

27 l'Union turque de Xanthi et l'Union des Jeunes Turcs de Komotini ont èté créées à la fin des années 1920, sur le modèle des structures de diffusion du kemalisme existant en Turquie. Ces associations n'ont malheureusement jamais fait l'objet d'une étude en soi, et les seules informations disponibles (et seulement pour la période de l'entre-deux-guerres) apparaissent dans la thèse de Vemund Aarbakke (voir note 25).

${ }^{28}$ Un de nos interlocuteurs, membre fondateur du réseau associatif et ancien président de l'association de Düsseldorf, affirme que c'est lui qui avait été élu à la tête de la Fédération en 1988. Son rival, Cafer Alioğlu, lui aurait téléphoné en lui annonçant qu'il avait lui-même été désigné comme président, et qu'il s'agissait "d'ordres venus d'en haut " (entretien à Viersen, mars 2001). 
fêtes nationales turques, notamment la " fêtes des enfants " qui commémore la séance inaugurale de l'Assemblée nationale turque le 23 avril 1920. Mais il ne s'agit pas là d'un phénomène généralisé, et nombre de nos interlocuteurs en Allemagne n'ont qu'un rapport lointain à la Turquie ${ }^{29}$, se dissociant clairement des Turcs de Turquie dont ils ne veulent pas dans l'Union européenne.

Ainsi, le soir du kurban bayramı (fête du mouton) à Witten, en 2003 , regardant les informations télévisées des chaînes de télévision turques, nos hôtes - le président de la Fédération et sa famille - ne cachaient pas leur dégoùt devant les scènes d'abattage collectif ou illégal dans les rues d'Istanbul. " Les Turcs sont sales et ignorants " est un jugement péremptoire qui fait l'unanimité parmi nos interlocuteurs, s'appliquant aussi bien aux habitants de la Turquie qu'aux ressortissants turcs en Allemagne, qui seraient responsables de la crise économique que traverse le pays ${ }^{30}$. Les hommes de la première génération de migrants (nous verrons que les femmes s'interdisent généralement tout discours à caractère politique) sont plutôt critiques vis-à-vis de la Turquie moderne qui n'a pas, selon eux, respecté les principes laïques édictés par Mustafa Kemal, ce qui expliquerait l'émergence d'une mouvance islamiste. Cela n'empêche pas que, pour ceux d'entre eux qui ont, ou ont eu, des responsabilités associatives, le fait d'être invité ou de participer à des manifestations (colloques et festivals) relatives aux Turcs de Thrace occidentale en Turquie est considéré comme un honneur, comme l'assurance du renforcement de leur prestige au sein du groupe.

Et puis il y a ceux qui, au contraire, militent pour les idéaux du nationalisme turc, comme le président de l'association munichoise (voir infra), ou le concierge de l'association des Furcs de Thrace occidentale à Bursa, qui a vécu trente-cinq ans en Allemagne où il militait au sein du MHP ${ }^{31}$. Ces trajectoires de vie différenciées ont le mérite de faire ressortir l'hétérogénéité du groupe, et le fait qu'il n'existe pas de lien mécanique entre la turcité revendiquée d'une part, l'identification à la Turquie et/ou l'allégeance au nationalisme turc d'autre part. En revanche, le fait qu'il existe une stratégie européenne de l'État turc pour préserver ses intérêts en Thrace occidentale permet de nuancer le constat

\footnotetext{
${ }^{29}$ La grande majorité de nos interlocuteurs, en Grèce comme en Allemagne, a soit de la famille, soit une résidence secondaire (voire les deux) à Bursa, la quatrième ville de Turquie dont $9 \%$ de la population est originaire des Balkans. Ces résidences secondaires, acquises du temps oủ tout investissement immobilier était interdit aux musulmans en Thrace, sont souvent vides ou louées. Pour nombre de nos interlocuteurs, en effet, la Turquie n'est qu'un lieu de passage.

$3^{\circ} \mathrm{Ce}$ genre de réactions méprisantes se retrouve aussi en Turquie, au sein de l'intelligentsia et de ceux qu'on appelle les "Turcs blancs", pour qui les Turcs et Kurdes issus de l'exode nural ont " envahi " et " sali " Istanbul. A ce propos, voir Bozarslan (Hamit), Histoire de la Turquie contemporaine, Paris : La Découverte, 2004, p. 85.

${ }^{31}$ Parti d'action nationaliste (Milliyetçi Hareket Partisi) fondé par Alparslan Türkeș, qui faisait partie de la coalition gouvernementale au pouvoir en Turquie avant les élections législatives de novembre 2002.
} 
selon lequel " la Turquie accorde sans doute moins d'importance aux Balkans qu'à ses rapports avec les pays occidentaux ou à l'élargissement de sa sphère

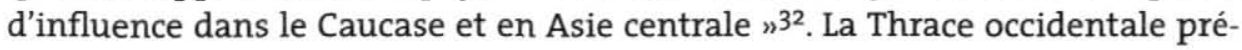
sente en effet la particularité pour la Turquie d'accueillir la seule population "turque" et européenne.

\section{RAPPORTS DE GENRE, LIEUX DE SOCIABILITÉ ET TRANSMISSION DE L'IDENTITE DU GROUPE}

\section{L'existence d'un discours politique sur la famille}

Nous allons voir maintenant la structuration interne des associations, et le lien qui peut être fait entre cette organisation et la transmission de l'identité du groupe. Nombre d'associations sont organisées sur le même modèle et comportent trois sections : celle des hommes, celle des femmes et celle des jeunes 33 . Plusieurs associations ont également un club de football et un groupe de danse folklorique. Il s'agit là non seulement d'une logique de perpétuation identitaire, à travers un processus de folklorisation, mais également d'une stratégie d'encadrement des individus. Nos interlocuteurs insistent en effet sur le fait que les activités de l'association, et particulièrement le sport, permettent aux jeunes de se rassembler au sein de l'association, et les protègent ainsi de l'oisiveté, du fait de "traîner dans les rues ", et des mauvaises fréquentations. Ces activités permettent en outre aux jeunes Turcs de Thrace de se rencontrer entre eux, en ne se mêlant pas trop aux jeunes Allemands, dont le mode de vie est souvent jugé peu recommandable, en référence à la consommation d'alcool et à la liberté sexuelle des jeunes femmes. L'organisation associative, basée sur la division sexuelle des tâches et des espaces, révèle notamment un effort de transposition du modèle familial et domestique, avec ses hiérarchies et ses espaces de vie différenciés.

La famille est la base de l'organisation sociale, mais elle est aussi érigée en valeur, garante de l'ordre social et de la pérennité du groupe, et parfois même en valeur " nationale ». Comme l'écrit M.Özkan, président de l'Union des familles turques de Thrace occidentale de Bavière (Bavyera Eyaleti Batı Trakya Türkleri Aile Birliği), dans un manuel largement diffusé en Allemagne : « Voici

\footnotetext{
${ }^{32}$ Bougarel (Xavier), Clayer (Nathalie), éds., Le nouvel islam balkanique. Les musulmans, acteurs du postcommunisme (1990-2000), Paris : Maisonneuve \& Larose, 2001, p. 45.

33 Le cloisonnement hommes/femmes intervient après le mariage, la section des jeunes ayant de façon implicite vocation à favoriser et encadrer les rencontres amoureuses, de même que les gece (soirées dansantes).
} 
quelques uns des éléments les plus importants qui maintiennent debout une nation : a) l'unité religieuse, b) l'unité linguistique, c) l'unité historique, d) l'unité culturelle et familiale " 34 . Cette association est la structure la plus importante au sein de la Fédération. Fondée en 1981 à Munich, elle regroupe depuis 1996 les associations de Nuremberg, Lauf, Herzogenrauch ; elle gère un club de football, un groupe de danse folklorique. Depuis 1988, une mosquée a été aménagée dans un bâtiment loué par l'association, reconverti à cet effet. Cette " mosquée de Thrace occidentale " (Batı Trakya Camii) sert également de lieu de culte aux fidèles munichois originaires de Turquie ; des cours de religion et de " culture turque " y sont dispensés 35 .

Dans la mesure où peu de nos interlocuteurs tiennent un discours semblable à celui de $M$. Özkan, on peut se demander ce qui, hormis le référent partagé de la turcité, homogénéise le groupe. Les divergences politiques, quoique moins marquées que dans les années 1970, sont bien réelles et affleurent à travers le rapport des acteurs à la Turquie officielle. Il semble que ce soit les codes sociaux et les valeurs familiales qui établissent le consensus nécessaire au maintien du groupe, ainsi que l'existence d'une figure mythique transcendant les clivages politiques, celle du Dr. Sadık Ahmet. Celle-ci constitue un symbole suffisamment fort pour éteindre toute contestation : critiquer l'illustre défunt, c'est s'exclure du groupe. En outre, les rumeurs de nature politique ou les commérages sur la vie privée sont, comme nous le verrons, un bon moyen de maintenir l'ordre et les frontières du groupe.

\section{La prégnance des rôles sexués dans l'organisation sociale du groupe}

Ce sont surtout les hommes qui fréquentent les associations, principalement en fonction de critères de génération - comprise ici comme communauté d'expérience et non comme classe d'âge - et de capital culturel. Dans certaines villes comme Berlin ou Viersen, le local associatif a été investi par les hommes issus de la seconde vague migratoire, qui ont remplacé les primo-arrivants. L'homme qui a présidé la Fédération jusqu'en 2004 est un architecte qui a

\footnotetext{
34 Özkan (Hüseyin), Teşkilât ve İdare (Organisation et Administration), München / İzmir : Bavyera Eyaleti Batı Trakya Türkleri Aile Birliği / Batı Trakya Araștırma Merkezi Yayınlan, 2002 [200o], pp. 143-144. Il s'agit là d'une terminologie caractéristique de la rhétorique du MHP et de son fondateur Alparlsan Türkeș. Nous remercions Hamit Bozarslan d'avoir attiré notre attention sur ce point. Cet ouvrage se veut être un "manuel" (el kitabl), comme l'indique son sous-titre, proposant un mode d'emploi pour l'activité associative : il fait une large place à l'organisation interne des associations, notamment au rôle dévolu à la section des femmes. L'ouvrage se veut aussi un mode d'emploi idéologique : il comporte un lexique expliquant entre autres ce qu'est un vrai chef, ce qu'est un bon nationaliste, et dénonçant le communisme.

35 Nous n'avons pas mené d'entretien dans cette partie de l'Allemagne, les informations suivantes sont issues des mémoires de Alioğlu (Cafer), op.cit.
} 
grandi en Allemagne, à ce titre il ne se sent pas vraiment proche des membres de l'association de sa ville, Witten. Il n'aime pas s'y rendre, bien que son mandat l'oblige à des visites régulières : il estime avoir mieux à faire que de " jouer aux cartes en regardant la télévision".

Les enfants des primo-arrivants dans l'ensemble fréquentent peu les associations ; ceux qui le font s'y investissent entièrement (comme à Gütersloh, voir infra). On croise rarement des femmes dans ces associations; néanmoins certaines structures ont créé une section féminine, qui organise des activités séparées, tels les cours de broderie, tissage et peinture sur soie visant à recréer le folklore de la Thrace, ou bien les " journées de femmes " (kadm günleri) au cours desquels le local associatif leur est réservé ; elles s'y réunissent et partagent les plats qu'elles ont cuisiné.

Lors des rares occasions où hommes et femmes se retrouvent ensemble à l'association, une division sexuelle de l'espace apparait. Ainsi, dans le local de l'association berlinoise où plusieurs membres avaient été convoqués par le président en notre honneur, un des hommes fit remarquer qu'il n'était pas convenable pour les hommes d'aller s'asseoir à la table des femmes. Cette séparation physique ne fait que refléter un mode de socialisation et d'organisation des rapports entre sexes en vertu duquel les attributions et compétences masculines et féminines sont strictement différenciées et intériorisées. Ce sont les hommes qui " parlent politique ", les femmes ne participent pas, ou rarement (là encore une distinction est à faire entre les différentes générations) à ces conversations, et elles s'en excluent d'elles-mêmes.

Dans et hors cadre associatif apparait ce qu'on pourrait appeler un " discours de genre " : les revendications des Turcs en Thrace occidentale sont systématiquement exposées par les hommes, quasiment jamais par les femmes. Lorsqu'elles le font, elles s'en remettent généralement au jugement plus sûr des hommes ${ }^{36}$. Les femmes ont du coup, paradoxalement, une parole plus libre. Alors que le discours dominant parmi les "Turcs " de Thrace - et particulièrement au sein du réseau associatif - revendique l'identité turque de l'ensemble de la minorité, la femme du président de la Fédération et sa jeune sœur plaisantaient au sujet d'une famille de Witten, originaire d'Ehinos (Șahin), que nous avions rencontrée la veille : "Alors, tu as rendu visite aux Anglais ?" avant de poursuivre, "ils refusent qu'on les appelle "Pomaques" et le prennent comme une insulte, alors on dit "les Anglais" quand on parle d'eux "37. Cette re-

${ }^{36}$ Le leitmotiv selon lequel " les femmes ne savent pas "a èté relevè en d'autres circonstances et dans d'autres "aires culturelles". Voir Centlivres (Pierre), Centlivres-Demont (Micheline), "Pratiques quotidiennes et usgaes politiques des termes ethniques dans l'Afghanistan du Nord-Est ", in Digard (JeanPierre), èd., Le fait ethnique en Iran et en Afghanistan, Paris : Edjtions du CNRS, 1988, p. 240.

37 Entretien réalisé à Witten, février 2003 . 
marque reflète le fait que, en Thrace comme en Allemagne, les catégories "Pomaques", “Tsiganes", "Turcs"38 continuent de faire sens dans les représentations du sens commun.

\section{Transmission de l'identité, marché matrimonial et prestige social}

À travers l'étude des associations comme lieu de prise en charge des membres du groupe, c'est également la question de la transmission de l'identité et des normes propres à celui-ci qui se pose. Les enfants des primo-arrivants que nous avons rencontré dans le cadre de ces enquêtes ont aujourd'hui entre 20 et 30 ans ; ils ont dans l'ensemble été peu sensibilisés par leurs parents à la situation politique en Thrace occidentale. Ainsi, les deux filles du président de l'association berlinoise nous demandèrent de leur expliquer pourquoi et comment les Turcs sont devenus une minorité en Thrace occidentale. Bien que leur père se soit toujours investi dans la vie associative ${ }^{39}$ et qu'il ait milité pendant de nombreuses années au sein du Parti Communiste grec (KKE), elles n'ont hérité ni sa culture militante, ni son savoir sur la Thrace occidentale. Elles n'ont pas été insérées dans un tissu social composé de migrants originaires de Thrace occidentale, peu nombreux à Berlin, et se désintéressent totalement de l'association où elles vont le plus rarement possible.

La belle-sœur du président de la Fédération a elle aussi grandi à l'écart des autres Turcs de Thrace ; elle dit n'avoir su parler le turc correctement qu'à l'âge de dix-sept ans $4^{\circ}$. Ses parents participaient peu aux activités de l'association, si ce n'est aux pique-niques, où on ne parlait pas de la Thrace mais de choses du quotidien. Fréquentant peu les "soirées familiales " (aile gecesi), cette jeune fille n'a pris conscience de la question de la Thrace occidentale que lorsque sa sœur a épousé celui qui allait devenir le président de la Fédération. On peut alors identifier les facteurs permettant ou facilitant la transmission de la mémoire de cette population en tant que minorité : le type de rapports entretenus par les membres d'une famille (les jeunes filles de Berlin ont honte de leur père qui parle très mal l'allemand), la possession d'un certain capital culturel et social, la présence dans l'entourage d'autres membres du groupe, surtout s'ils sont actifs au sein des structures associatives.

Essayons maintenant de comprendre pourquoi et comment les aile gecesi sont un élément central du dispositif de transmission identitaire et de politisation. Quoique connaissant assez peu l'histoire de la " minorité turque de

\footnotetext{
${ }^{38}$ Dans ce cas, il est souvent fait allusion au fait d'être ou pas un "vrai Turc n ou un " Turkmène ", ce qui réintroduit un niveau de distinction entre le fait d'être turc, associé à une revendication d'ordre politique, et le fait d'être "réellement" et "authentiquement" turc, avec une connotation racialisante.
}

39 C'est le seul de nos interlocuteurs à fréquenter parallèlement deux associations, l'une grecque l'autre turque, en plus de celle qu'il a créée pour les Turcs de Thrace.

$4^{\circ}$ Entretien réalisé à Komotini, août 2002. 
Thrace occidentale ", nos jeunes interlocutrices savent en revanche parfaitement comment se comporter en présence de leur famille et d'autres membres du groupe, particulièrement lors des gece (fêtes), qui sont par excellence des lieux de représentation sociale. Les fêtes organisées par les associations sont en effet des lieux de sociabilité et de contrôle social, mais aussi de politisation. L'élégance y est de rigueur (sauf pour les femmes mariées portant le voile : elles ne dansent pas et ne quittent pas le long manteau qui les enveloppe jusqu'aux chevilles) ; le déroulement de la soirée est convenu et les comportements strictement codifiés. Les cigarettes et l'alcool sont réservés aux hommes ; les femmes fument dans les toilettes, qui deviennent dès lors des espaces de sociabilité féminine. Nos jeunes interlocutrices se plient à ces règles ou les contournent habilement (par exemple en buvant de l'alcool dans des verres dont on ne peut voir le contenu), car elles connaissent le risque encouru : le dedikodu, ou commérage, qui entamerait la respectabilité de leurs parents.

Ces soirées constituent également une sorte de marché matrimonial, un espace de rencontre encadré puisque les jeunes hommes et femmes sont accompagnés de leur famille. Cela n'est pas sans importance pour un groupe qui prône l'entre-soi comme valeur de référence : il vaut toujours mieux épouser un Turc de Thrace occidentale plutôt qu'un Turc de Turquie, et le fait d'épouser un Allemand peut impliquer pour une jeune fille d'être bannie de sa famille ${ }^{41}$. Cela est également valable pour les hommes, mais de façon plus souple, sans conséquence aussi irrémédiable. Dans un deuxième temps, les jeunes hommes et femmes peuvent prolonger la soirée entre eux, et aller en discothèque où ils pourront abandonner leur rôle d'enfants modèles et consommer librement de l'alcool. Néanmoins, ce type de sociabilité favorise la reproduction du groupe d'amis des parents vers les enfants ${ }^{42}$, surtout si l'on prend en compte le fait qu'aux liens d'amitié se superposent souvent, à des degrés divers, des liens de parenté.

Contrairement à ce que semble indiquer leur nom, les aile gecesi ne sont pas seulement des lieux de rencontre familiaux. Elles sont le lieu où se joue le prestige social, où se font et se recomposent les réseaux de personnalités de marque, comprenant les présidents d'association et membres du conseil d'administration (yönetim kurulu) de la Fédération. Les présidents des différentes associations représentées à la fête échangent leurs cartes de visite, et se prennent mutuellement en photo, par petits groupes : cela fait partie du rituel, la

\footnotetext{
${ }^{41}$ Un seul cas aussi extrème nous a été mentionné. De toute façon les jeunes filles - du moins celles qui ont ètè interrogées dans le cadre de cette enquète - sont conditionnées pour ne pas tomber amoureuses d'étrangers au groupe, ou au moins de non Turcs, ce qui limite ce type de drame. C'est pourquoi les séjours estivaux en Thrace constituent un temps fort de la sociabilité du groupe, en raison du potentiel ma. trimonial que constituent les Turcs de Thrace restés au pays.
}

$4^{2}$ Ruffier (Amaud), "Les Gap et Ziyâfat : reconstitution du passé à partir du regard d'un ethnographe soviétique sur une société centre-asiatique traditionnelle », CEMOTI, (34), 2002, p. 52. 
photo scelle chaque nouvelle amitié ou relation entre les acteurs associatifs et/ou politiques. Ces fêtes sont l'occasion pour les consuls de Turquie de venir rencontrer ces acteurs et d'identifier ainsi leurs interlocuteurs au sein du réseau associatif. Une sorte de rituel s'installe autour de ces rencontres, généralement immortalisée par une photo de groupe publiée ensuite dans l'édition allemande de journaux tels que Hürriyet ou Türkiye. Cet attachement à la photo est précieux pour reconstituer les parcours des acteurs, lorsqu'ils ouvrent leurs albums de famille : s'y trouvent immortalisées leurs rencontres, en Allemagne et en Turquie, avec des présidents d'association ou des hommes politiques. Les personnalités en vue sont, en effet, celles qui ont le réseau de connaissances le plus étendu, et qui sont vues en compagnie d'acteurs étatiques ou associatifs de Turquie. Les gece sont, enfin, un lieu d'expression politique.

\section{Les gece comme lieu d'expression politique}

Pour mieux comprendre le rôle politique - particulièrement affirmé dans les années 1980 - que peuvent avoir les aile gecesi, il convient de les distinguer des fêtes à caractère privé, réservées aux proches et plus particulièrement aux femmes et aux enfants, comme les fêtes de circoncision (sünnet) ou les kina gecesi (littéralement : " fête du henné ", qui clôt la vie de jeune fille de la future mariée). Cette typologie sommaire est inspirée de celle, plus complexe, établie par Arnaud Ruffier à propos de l'Ouzbékistan 43 L'auteur y opère une distinction entre des fêtes mobilisant différents réseaux de sociabilité 44 d'une part, et d'autre part entre les finalités de ces différents types de fêtes : celles, ouvertes, qui ont simplement vocation à rassembler les membres du groupe et à montrer son homogénéité, et celles à caractère privé, qui ont une vocation plus politique. De cette typologie élaborée pour étudier les modes de sociabilité et la dissidence dans un État autoritaire, on retiendra la démarche consistant à identifier ce qui relève de l'expression politique au sein de rassemblements festifs, donc rarement considérés comme des espaces de contestation et de politisation.

Un de nos interlocuteurs nous a raconté l'ambiance des aile gecesi de son enfance, au début des années 1980, qui rassemblaient jusqu'à 2 ooo personnes : les associations étant moins nombreuses, les fêtes étaient plus rares et dispersées. Étant le fils du président de l'association de Düsseldorf et ayant une bonne connaissance de la langue turque, contrairement à nombre de jeunes nés en Allemagne, il avait l'obligation lors de ces fêtes de monter sur scène pour déclamer des hymnes à la guerre d'Indépendance turque (1922-1923), mo-

43 Ibid.

44 Celles rassemblant l'ensemble des connaissances d'un individu, c'est-à-dixe " tous les cercles de sociabilité directs et indirects dans lesquels il est inséré $n$, et celles rassemblant seulement les personnes ayant " établi avec lui un type particulier de rapport ". Ibid., p. 57. 
ment fondateur de la mémoire nationale et de l'hagiographie kémaliste en Turquie. Un peu gêné, il précisa qu'à l'époque il ne comprenait rien à ce qu'il récitait, et que le choix de ces poèmes était lié au climat politique de l'après crise de Chypre en Thrace occidentale 45 .

Cela montre que dans le cadre fermé des aile gecesi (par opposition au cadre public de la société d'accueil et des institutions européennes), une parole plutôt radicale se libérait. On peut en déduire que les cadre juridiques et politiques allemand et européen influent sur le répertoire d'action choisi et sur les modes d'expression publique des revendications liées à la turcité, sans remettre fondamentalement en cause les représentations véhiculées par d'autres canaux, internes au groupe. L'anecdote nous donne ainsi une indication quant à l'encadrement des Turcs de Thrace occidentale d'Allemagne par les référents du nationalisme turc, visibles dans certaines associations à travers cartes, livres ou affiches de promotion des manifestations organisées en Turquie sur le thème du " monde turc ".

Ces référents constituent certes un univers de sens commun à tous, renforcé par les souvenirs et rumeurs concernant les brimades subies en Grèce, mais un univers de sens malléable et volatil qui contient l'hétérogénéité des trajectoires et représentations des acteurs associatifs. Le président de l'association de Düsseldorf d'il y a vingt-cinq ans, dont le fils déclamait des poèmes bellicistes et nationalistes, est également celui qui réprouve aujourd'hui la trop grande intégration du réseau associatif d'Allemagne à celui de Turquie. Il ne faut pas mésestimer la capacité qu'ont les acteurs de s'approprier ou de composer avec ces référents, surtout dans la mesure où ils sont la clé pour l'accès à des positions, sinon de pouvoir, du moins de prestige au sein du groupe. Ces référents constituent en somme un substrat de la mémoire collective, mais aussi un répertoire lexical et sémantique à maîtriser afin de se faire accepter comme représentant potentiel par le groupe.

La dimension politique des fêtes est toujours présente aujourd'hui, quoique sur un mode routinisé. Ainsi le bal du Nouvel An 2004 organisé à Dortmund par la Fédération, et ayant rassemblé 2500 personnes, s'est déroulé - à deux mois des élections législatives du 7 mars 2004 - en présence des deux députés turcs de Komotini (Gümülcine), élus du Parti socialiste (PASOK), et du président l'Union turque de Xanthi ${ }^{6}$. Les associations d'Allemagne sont en effet devenues un point de passage obligé pour les personnalités politiques de

\footnotetext{
45 Entretien réalisé à Düsseldorf, février 2003.

46 " Gurbetçilerin muhteșem bulușması " (La magnifique rencontre des "exilés"), Gündem, 02/01/O4. Gündem (" Ordre du jour n) est un hebdomadaire qui parait à Komotini depuis 1996. Il illustre deux changements au sein de la minorité : le retour au pays des jeunes partis faire leurs études en Turquie et/ou en Europe, ainsi que la professionnalisation de la presse locale. Comme plusieurs journaux turcophones locaux, il est subventionné par le consulat de Turquie.
} 
la Thrace : un mois après sa prise de fonction, lilhan Ahmet, seul élu de la minorité au Parlement grec depuis le 7 mars 2004, rendait visite à l'association des Turcs de Thrace occidentale de Bavière. Mais elles ne sont plus, comme dans les années 1980 et 1990, le relais privilégié auprès des institutions européennes d'un mouvement de protestation affaibli par la politique d'ouverture de l'État grec, et par la concurrence de la nouvelle génération d'acteurs associatifs en Thrace occidentale.

\section{LA RELIGION, ENTRE PRATIQUE SOCIALE ET RESSOURCE POLITIQUE}

\section{Les différences de pratiques à l'aune des trajectoires migratoires}

Nos enquêtes ayant pour objet l'usage politique du sacré et l'étude de la mobilisation politique par la voie associative, les pratiques religieuses ont pu être observées au quotidien, dans la sphère privée, mais n'ont pas fait l'objet d'un questionnaire spécifique. C'est pourquoi on est contraint ici à un certain niveau de généralité, notamment au sujet des pratiques masculines. Ce sont les femmes qui respectent les interdits communément associés à l'islam (concernant l'alcool et le tabac)47, surtout celles qui sont arrivées en Allemagne à l'âge adulte. Concernant les codes vestimentaires et le port du voile (désignant ici le fait de se couvrir les cheveux et le cou), les femmes voilées rencontrées au cours de ce travail de terrain (notamment à l'association de Berlin) étaient issues d'une immigration plus récente et rurale (région pomaque du nord de Xanthi). Pour ce qui est de la pratique religieuse proprement dite, ce sont principalement les femmes qui exécutent les cinq prières quotidiennes ; la pratique est toutefois peu répandue, elle a lieu à la maison car d'après les codes sociaux en vigueur, seuls les hommes vont à la mosquée. Les interdits et prescriptions alimentaires, ainsi que le jeûne du ramadan, sont en revanche plus largement observés, par les hommes comme par les femmes.

Les temporalités migratoires sont ici à prendre en compte. Parmi les primo-arrivants, la plupart de nos interlocutrices ont abandonné le port du voile à leur arrivée en Allemagne. C'est surtout vrai pour celles qui sont originaires des villages, la modernité citadine se traduisant en Thrace même par l'abandon du foulard. Le constat est différent pour la seconde vague migratoire, dans les années 1990 . Nombre de nos interlocutrices venues à cette pé-

\footnotetext{
47 Pour les raisons exposées ci-dessus, la question des pratiques sexuelles, et plus spécifiquement la prescription de la virginité des femmes au mariage, n'a pas été posée lors des entretiens, et n'est pas abordée ici. Si cette prescription est, de façon tacite, fortement ancrée, il est difficile de dire dans quelle mesure elle est respectée ou contournée.
} 
riode sont originaires de villages du département de Xanthi, elles portaient le voile avant leur arrivée en Allemagne et l'ont conservé. On assiste également chez certaines primo-arrivantes à un retour à la pratique religieuse. Le fait de porter le voile, voire de l'assortir d'une pratique assidue de la prière, peut en effet correspondre au passage d'un cycle de vie à un autre : devenir quadra ou quinquagénaire, ressentir un sentiment d'inutilité lié à une sortie de la vie active. On voit ainsi que la pratique religieuse et les codes vestimentaires liés à l'islam sont en grande partie une affaire de conventions sociales et une marque de respectabilité.

Nos interlocuteurs masculins ne fréquentent généralement pas la mosquée sauf à l'occasion des fêtes religieuses (notamment la fin du ramadan) ou d'événements qui marquent la sacralité du groupe (voir infra). Mentionnons néanmoins, en contre-exemple, une famille de Witten originaire d'Ehinos (Șahin), village situé au nord de Xanthi, dans l'ancienne zone militaire fermée. Cette famille est connue du président de la Fédération et de ses proches, qui vivent dans la même ville, mais ils n'entretiennent aucun contact : elle se singularise par le fait qu'elle fréquente la mosquée des " fondamentalistes " (selon le terme de nos interlocuteurs).

Alors que la grande majorité de nos autres interlocuteurs a conservé la nationalité grecque, les hommes de cette famille ont demandé la nationalité allemande pour leurs enfants : l'Allemagne, contrairement à la Grèce et à la Turquie, permet le port du voile à l'école et à l'université. Lors de notre rencontre, l'aîné des deux hommes a refusé de nous serrer la main, expliquant qu'il ne pouvait toucher une femme. Les convictions et pratiques religieuses de cette famille paraissent être le résultat de leur socialisation religieuse en Allemagne ; cela pose la question de la spécificité de la pratique religieuse des Turcs de Thrace occidentale. Une telle question fait intervenir toute une série de variables qui mériteraient en soi de faire l'objet d'une recherche. C'est pourquoi nous préférons insister sur les usages politiques du registre religieux dans le cadre de la mobilisation identitaire des Turcs de Thrace occidentale.

\section{L'usage de la religiosité en politique : la sacralisation de la figure de Sadık Ahmet}

Tout d'abord, précisons que les prêches constituent souvent, en Thrace comme ailleurs, l'occasion d'exprimer une opinion politique ${ }^{4}$, de même que les lieux de culte sont prisés en période électorale 49 . Le processus de sacralisation de la figure du Dr. Sadık Ahmet répond à une logique différente. La reli-

$4^{8}$ Comme le relate un reportage réalisé en septembre 2003, au moment du Miraç Kandili (célébration de l'ascension du prophète Muhammad au paradis). Voir " Meriç'in öte yakası : Batı Trakya Türkleri n (art.cit.), p.182.

49 "Batı Trakya'da heyecan " (La tension monte en Thrace occidentale), Milliyet, 29/02/04. 
gion sera ici considérée non pas dans son sens immédiat, en tant que système de croyances, de dogmes et de pratiques, mais plutôt en tant que référent émotionnel ayant une fonction cohésive et visant à conférer une nature sacrée à certains événements ou figures politiques. La religion comprise dans ce sens est proche de la " religiosité séculaire " (sekuler dinsellik) décrite par Birol Çaymaz ${ }^{50}$, elle a des référents universels. Nous allons ici nous intéresser à la façon dont la figure de Sadık Ahmet est mise en scène afin de lui conférer une aura mythique et héroïque.

Dans chaque association en Allemagne se trouve un portrait " officiel " de Sadık Ahmet, toujours le même, près de celui d'Atatürk, avec un drapeau de la République de Turquie, et parfois celui de l'éphémère République turque de Thrace occidentale, fondée en $1913^{51}$. D'ailleurs, si Sadık Ahmet incarne la figure du héros, l'épisode de la République de Thrace occidentale (souvent associé, voire confondu avec la période d'administration interalliée de la Thrace, sous le commandement du général français Charpy, en $1919^{52}$ ) symbolise la naissance de la Thrace en tant qu'entité politique autonome, antérieurement à son rattachement à la Grèce.

Les portraits de Sadık Ahmet sont fréquemment accompagnés d'une inscription : "Tu nous manques ", "Tu es parmi nous ", " Nous ne t'oublierons pas ". On trouve également l'expression " aziz Dr. Sadık Ahmet " qui signifie aussi bien " cher " que " saint ". Ces phrases ne sont pas sans rappeler les épitaphes des cimetières, aussi bien musulmans que chrétiens ; ou encore les évocations de Jésus dans la liturgie chrétienne, systématiquement associées à l'image, ce qui n'est pas le cas dans la tradition islamique. Cela n'est pas contradictoire avec le fait de se trouver face à un groupe dont les référents sont ceux de l'islam : " N'existe-t-il pas un socle commun aux usages religieux et civils de la figure du martyr (ou plus largement du "grand mort") qui détermine ou oriente leurs effets sociaux indépendamment de la position d'un horizon religieux préalable ? "53.

\footnotetext{
$5^{\circ}$ Birol Çaymaz a de la même façon analysé les procédés utilisés pour fidéliser le peuple turc à la jeune République de Turquie : bien que la religion ait été reléguée à la sphère privée, la religiosité (dinsellik) est constamment utilisée, surtout pour la sacaralisation de certains personnages, à commencer par Atatürk. Dans la production littéraire officielle de l'époque, l'amour porté au Créateur (Dieu, yaratici) est remplacé par l'amour au Gâzi créateur (le terme est le même). Çaymaz (Birol), " Le $10^{\text {ème }}$ anniversaire de la République de Turquie ", exposé présenté dans le cadre du séminaire d'Alexandre Toumarkine Rites politiques de la Turquie républicaine (1923-1938), Institut français d'études anatoliennes, Istanbul, 29 avril 2004 .

${ }^{51}$ Les portraits de Sadik Ahmet, tout comme le drapeau de la République de Thrace occidentale, sont plutôt absents des locaux associatifs visités en Thrace, alors qu'ils sont omniprésents en Turquie. En Thrace, les symboles de l'amitié gréco-turque sont privilégiés : les drapeaux grec et turc, ainsi que les portraits d'Atatürk et Venizelos rappelant le traité d'amitié entre les deux pays, signé en 1932.
}

52 Dalègre (Joëlle), «La mission du général Charpy en Thrace occidentale», Mesogeios, (4), 1999.

53 Albert (Jean-Pierre), " Sens et enjeux du martyre : de la religion à la politique ", in Centlivres (Pierre), éd., op.cit., p. 22. 
Cette liturgie semble pourtant limitée à l'espace associatif : on trouve rarement un portrait de Sadık Ahmet dans l'espace domestique, sauf chez le président de la Fédération, alors que celui d'Atatürk y est souvent présent. On peut émettre l'hypothèse que la disparition de Sadık Ahmet est encore trop récente pour que le processus d'objectivation de son caractère mythique et héroïque soit achevé, comme c'est le cas pour Atatürk à qui le culte rendu confine au " consensus obligatoire " 54 .

Le personnage de Sadık Ahmet a encore un caractère trop polémique et, passés les éloges d'usage et la satisfaction liée au fait d'avoir côtoyé ce grand homme, ceux de nos interlocuteurs qui ont travaillé avec lui lors de ses premiers séjours en Allemagne évoquent les désaccords suscités par son discours extrêmement virulent. Ainsi, l'ancien président de l'association de Düsseldorf finit par exprimer ouvertement son amertume : seul Sadık Ahmet, de son vivant, bénéficia des retombées et du prestige de la mobilisation des associations d'Allemagne, dont il fut lui-même l'un des principaux acteurs 55 .

\section{La production d'événements autour de la figure mythique}

Le rôle politique de la figure de Sadık Ahmet est néanmoins évident. Des événements ont ainsi été " créés ", comme ceux du 29 janvier et du 25 juillet dont les commémorations se déclinent en Thrace, en Turquie et en Allemagne. Elles participent du " culte du héros " qui, " comme celui des saints, se nourrit d'un rapport organisé au temps et au lieu ${ }^{56}$. De telles commémorations se veulent des sortes de fêtes nationales, qui ponctuent la temporalité du groupe en créant des occasions d'échanges et de rencontres, et renforcent le sentiment d'appartenance identitaire.

La première de ces dates correspond à deux événements : les émeutes de 1988 à Komotini (Gümülcine), après que la Haute Cour de Grèce ait décidé d'interdire les associations portant le mot " turc " dans leur intitulé, et la manifestation organisée deux ans plus tard jour pour jour, afin de protester contre la condamnation en justice de Sadık Ahmet57. Quant à la seconde date, il s'agit de celle de la disparition de Sadık Ahmet, le 25 juillet 1995, dans un accident de la route qui a suscité la colère et la suspicion au sein de la minorité. L'enquête sur la collision qui a provoqué la mort de Sadık Ahmet a conclu à l'accident, mais

54 Copeaux (Etienne), "Le consensus obligatoire ", in Rigoni (Isabelle), éd., Turquie : les mille visages, Paris : Syllepse, 2000.

55 Nous avons rencontré cet interlocuteur à plusieurs reprises, en mars 2001 à Viersen, en août 2002 à Komotini, puis de nouveau à Viersen en février 2003.

${ }^{56}$ Mayeur-Jaouen (Catherine), " Grands hommes, héros, saints et martyrs : figures du sacré et du politique dans le Moyen-Orient du XXème siècle n, in Mayeur-Jaouen (Catherine), éd., op.cit., p. 28.

57 Il lui était reproché d'avoir fait campagne, lors des élections législatives de 1989 , sur le thème de la turcité de la minorité. 
ce brutal décès alimente le mythe du martyr, dont la rumeur prétend qu'il aurait été "éliminé " par les autorités grecques.

La réinterprétation des événements confère deux significations à la date du 29 janvier : la " résistance nationale " (millî direniș) en 1988, et la résistance aux " tentatives pour broyer la conscience de la turcité " (Türklük bilincini ezme çabalarl) en $1990^{58}$. Chaque année, à cette occasion, des conférences sont organisées par les associations d'Allemagne. Les intervenants en sont les personnalités politiques de la Thrace (qui se prévalent du combat mené aux côtés de Sadik Ahmet) et les dirigeants de l'Association de Solidarité des Turcs de Thrace occidentale en Turquie (Batı Trakya Türkleri Dayanısma Derneği - BTTDD), ces derniers étant auréolés de l'aura de la "mère-patrie " et de titres universitaires, ce qui leur confère un indéniable prestige ${ }^{59}$. En revanche, l'événement n'est pas commémoré en Thrace occidentale, alors qu'il l'était du vivant de Sadık Ahmet ${ }^{60}$ : là encore, c'est la volonté d'apaisement vis-à-vis des autorités grecques qui prévaut.

La disparition de Sadık Ahmet fait l'objet d'une commémoration officielle chaque année à Komotini. Des représentants des partis de la droite nationaliste et islamiste, ainsi que des hommes d'affaires, viennent chaque année de Turquie pour assister à la cérémonie au cimetière de Komotini (Gümülcine) ${ }^{61}$. Cette cérémonie est suivie d'une réception organisée par les personnalités politiques locales. Il s'agit plus d'une cérémonie à caractère fermé à laquelle seules participent les personnes " qui comptent ": la famille du défunt, les notables et les acteurs impliqués dans le mouvement identitaire turc (journalistes de la presse turcophone, membres actifs des associations turques). Le compterendu qu'a fait le journal Gündem de cette cérémonie en 2003 met particulièrement en valeur le culte du héros.

L'aspect strictement religieux de la cérémonie - une lecture de prières à la mosquée - est relaté en une phrase à la fin de l'article. En revanche, une des

\footnotetext{
${ }^{8} 8$ Batı Trakya Türkleri Dayanışma Derneğ̈, Batı Trakya neresi ? Batı Trakya Türkleri için 29 Ocak nedir ? (Oủ se trouve la Thrace occidentale ? Que signifie le 29 janvier pour les Turcs de Thrace occidentale ?), Istanbul : BTTDD, 2001.

59 Un ancien président de la BTTDD, alors vice-président du Centre de recherche de la Conférence islamique (IRCICA), avait été invité à une conférence en Allemagne à l'occasion du 29 janvier 2002. Il rous raconta son séjour avec beaucoup de fierté, sur un ton lègèrement paternaliste : dans toutes les associations qu'il avait visitées, il avait été accueilli avec beaucoup d'égards, et avait été touché du soin manifeste que les hommes avaient apporté à leur tenue en son honneur (entretien réalisé à lstanbul, février 2002).

60 "Batı Trakya'da 29 Ocak Türklük bayramı kutlandı " (La fête de la turcité a été célébrée en Thrace occidentale), "Dr. Sadik Ahmet'in 29 Ocak mesajı " (Le message du Dr. Sadık Ahmet pour le 29 janvier), Batr Trakya'nm Sesi, (46), janvier 1993.

${ }^{61}$ Depuis 2004 on observe un changement notable : ce ne sont pas les représentants de la droite nationaliste turque, mais des représentant du parti au pouvoir AKP qui ont assisté à la cérémonie, dont le députe de Bursa Mustafa Dündar, originaire de Komotini. Un tel changement est significatif mais son analyse déborde le cadre de la présente étude.
} 
photographies illustrant l'article montre la mère du défunt entourée d'hommes politiques et du représentant de la Chambre de commerce d'Ankara ${ }^{62}$. Avec son çarșaf (voile) blanc, elle évoque la figure d'une sainte, ce qui est a priori l'effet escompté. L'article consacre en outre une large place à la lecture d'une lettre de la fille de Sadık Ahmet à son père, au cours de la cérémonie du cimetière. Or, comme le rappelle Catherine Mayeur-Jaouen : " le moment des obsèques est souvent décisif pour la sanctification du défunt. [...] Après cette première étape, la commémoration de la mort du saint ou du héros est décisive pour la survie du culte ${ }^{63}$.

En Allemagne, selon la même source, la cérémonie avait un caractère religieux bien plus prononcé : il s'agissait d'une lecture de passages du Coran organisée à la mosquée de Witten. Le recours au registre religieux peut s'expliquer par l'absence de personnalité politique d'envergure qui aurait donné du lustre à la commémoration. Cela indique, de la part des dirigeants de la Fédération, une volonté de ne pas être en reste dans la dévotion à Sadık Ahmet, malgré leur position excentrée - éloignée de l'épicentre symbolique (la Thrace, enjeu de la mobilisation), et politique (la Turquie où est défini le répertoire d'action). En effet, même si les associations de Turcs de Thrace occidentale d'Allemagne jouent un rôle dans la constitution d'un imaginaire lié au gurbetçi (" exilé ")-le journal Gündem, par exemple, ne manque pas de relater les fêtes, manifestations culturelles et visites de personnalités de Thrace en Allemagne - leurs dirigeants ne peuvent prétendre avoir un rôle politique ${ }^{64}$. Même le "tournoi de football Sadık Ahmet ", que la Fédération organisait chaque année en Allemagne depuis 1996, se déroule en Thrace depuis 2003.

Enfin, dans le dispositif de culte du héros, le " Prix Sadik Ahmet pour la paix et l'amitié " (Dr. Sadık Ahmet Dostluk ve Barș Ödülü) mérite d'être mentionné, même s'il s'agit d'une initiative des associations de Turcs de Thrace occidentale de Turquie. Créé en 1996, il a été notamment décerné ces dernières années à la mère et à l'instituteur de Sadık Ahmet, à Panayote Dimitras, dirigeant de la section grecque de la Fédération internationale d'Helsinki pour les droits de l'homme, à Rauf Denktaș, ancien président de la République turque de Chypre du Nord et membre d'honneur de la BTTDD, au général d'état-major

62 La Chambre de Commerce d'Ankara et les "Foyers turcs" (Türk Ocaklan, de tendance nationaliste) ont organisé un colloque en hommage à Sadik Ahmet, dont les actes ont été publiés. Voir Kahramanyol (Mustafa), ed., Milletten Azmlğa Yolculuk. Dünü, Bugünü ve Geleceḡi ile Batı Trakya Türklügü (Le chemin du millet à la minorité. La turcité en Thrace occidentale, hier, aujourd'hui et dans le futur), Ankara : ATO. 2003 .

63 Mayeur-Inonen (Catherine), art.cit., p. 30.

64 En août 2003, Gündem relate l'initiative de quelques dirigeants de la Fédération, venus à Komotini rencontrer les personnalités politiques grecques locales pour " les féliciter de leur (ré)élection " aux élections d'octobre 2002. L'objectif semble avoir été de s'affirmer comme interlocuteur, et donc comme acteur politique, mais les démarches sont restées vaines, les personnes sollicitées étant en vacances, ou ayant jugé ces visiteurs peu dignes d'intérêt. 
Ismail Hakkı Karadayı, et à Sema Pişkinsüt, présidente de la Commission du Parlement turc pour les droits de l'homme.

La fonction cohésive de l'émotion doit ici être soulignée. Hormis leur constant rappel lénifiant aux valeurs de la paix et de l'amitié, qui reprennent presque à l'identique le nom du parti fondé par le défunt ${ }^{65}$, les comptes-rendus des cérémonies et manifestations en l'honneur de Sadlk Ahmet insistent sur la description des personnalités qui pleurent; ses mère, femme et enfants sont en général au premier plan de ces rituels, afin de témoigner de leur nostalgie du défunt ${ }^{66}$. Les " compagnons de route " de Sadık Ahmet bénéficient aussi, par ricochet, d'une gloire posthume. Ainsi, lors de sa visite en Allemagne après son élection, le député İhan Ahmet a participé à une cérémonie en mémoire de Mustafa Hafiz Mustafa, décédé en 1996. Celui-ci était le propriétaire de la revue Yuvamı (" Notre foyer ") à Komotini (Gümülcine), et avait fondé en 1992 avec Sadik Ahmet le journal du DEB (DEB Partisi Gazetesi), devenu ensuite Balkan. À cette occasion, l'association a élu sa femme, Fatma Hafız Mustafa, mère de l'année $2004^{67}$, jouant une nouvelle fois sur le registre familial.

\section{CONCLUSION}

L'objet de recherche Turcs de Thrace occidentale comporte une forte charge politique qui envahit les cadres de perception des acteurs, comme parfois des chercheurs eux-mêmes. Notre intention, dans cet article, était de montrer que ce n'est pas l'éventuelle allégeance à l'État turc qui structure le groupe. Comme dans d'autres cas, les réseaux et liens de parenté sont les ferments d'une configuration sociale, elle-même porteuse et productrice de normes de comporte-

\footnotetext{
65 Parti de l'amitié, de l'égalité et de la paix (Dostluk, Eșitlik ve Barş Partisi-DEB). Cet intitulé n'est pas sans rappeler celui des différents avatars de la mouvance islamiste en Turquie, successivement interdits : Parti de la prospérité (Refah Partisi), de la vertu (Fazilet Partisi), puis du bonheur (Saadet Partisi, toujours en activité). En vertu du principe de laicité, dont l'armée s'est érigée en garante, ces partis adoptent un nom se référant à des valeurs consensuelles, sans connotation politique. De la même façon, en Grèce le systèrne politique ne permet pas la formation de partis représentant les minorités, ce qui a conduit les fondateurs du DEB à adopter le même procédé.

66 "Gözyaşlan sel oldu " (Leurs larmes ont formé un torrent), " Valinin gözyașlan ”(Les lames du prèfet) sont les titres des paragraphes de l'article "Sadık Ahmet'i bir kez daha andık " (Nous avons célébré Sadık Ahmet une fois de plus), Batı Trakya'nın Sesi, (99), janvier 2002. Voir également "Bülent Aurnnç ağladı " (Bülent Annç - président du Parlement turc - a pleurê), Cumhuriyet, 19/04/03.

67 "Milletyekili ilhan Ahmet'in Almanya temaslan " (Prises de contact du député than Ahmet en Allemagne), Gündem, 16/04/04. Précisons que la désignation d'une " mère de l'année " fait partie des recommandations d'Özkan Hüseyir concernant la place et le röle des femmes dans les associations. Hüseyin (Özłan), op.cit.
} 
ment et de cadres de perception ${ }^{68}$. Le discours politique et/ou identitaire a également une vocation fédératrice, mais se situe à un autre niveau, qui est celui de la construction d'une " communauté imaginée "69.

Du point de vue des pratiques sociales, les différents exemples présentés montrent que l'on n'a pas affaire à un groupe homogène, pas plus en Thrace occidentale que dans l'espace migratoire. Les lignes de fracture ne sont pas tant les différentes composantes "ethniques" de la dénommée minorité musulmane, que les différents contextes sociaux (l'importance du clivage urbain/rural doit à cet égard ètre soulignée), trajectoires et socialisations. Cela est particulièrement vrai dans le cadre de la migration, qui suscite de nouveaux clivages et recompositions.

Nous avons également essayé de faire apparaître les mécanismes sur lesquels repose la mobilisation du groupe par les "entrepreneurs" associatifs. En Thrace occidentale, en tant que "minorité", ou en Allemagne en tant que "communauté étrangère", l'enjeu de la mobilisation n'est pas seulement d'ordre politique : il concerne la pérennisation du groupe, dont les rythmes et les règles de vie au sein de la sphère privée, mais aussi les modes d'expression publique, doivent s'adapter à ceux de la société d'accueil, avec lesquels ils interagissent. De ce point de vue, la baisse de fréquentation des associations - le président de la Fédération parlait d' "hémorragie " $70^{\circ}$ est à mettre en rapport avec l'insertion progressive des migrants et de leurs descendants dans la société d'accueil. Une analyse plus fine pourrait être menée, sur l'appropriation de l'espace associatif par les travailleurs issus de la seconde vague migratoire, à la fin des années 1980, comme c'est le cas à Berlin et Witten.

Létude de la prise en charge de cette population, en Allemagne, par les institutions gérées par des représentants de l'État turc, pourrait constituer un autre angle d'approche : l'école, où les élèves turcs ont la possibilité d'avoir des cours de "langue et civilisation" dispensés par des instituteurs venus de Turquie $^{71}$, et les mosquees, dont certaines sont gérées par la branche allemande de la Diyanet (Bureau des affaires religieuses). Dans le cadre de ce travail de terrain, on l'a vu, le domaine religieux a été considéré à travers les pratiques observées, en tant qu'élément des pratiques sociales. S'intéressant aux trajectoires migratoires, cette recherche n'a pas accordé de place spécifique

${ }^{68}$ Goffman (Brving), Les cadres de l'expérience, Paris : Minuit, 1991.

69 Anderson (Benedict), Imagined Communities. Reflections on the Origin and Spread of Nationalism, London / New York : Verso, 1983 .

$7^{70}$ Entretien réalisé à Witten, février 2003.

$\pi$ Ałgönül (Samim), " L'Etat turc et les Turcs européens : une tentative permanente d'encadrement paternaliste " in Dumont (Paul), Pèrouse (Jean-François), de Tapia (Stéphane), Akgönül (Samim), Migrations et mobilités internationales : Ia plate-forme turque, Les Dossiers de l'IFEA, décembre 2002, p. 85 . 
aux lieux de culte. Étant donné les multiples connexions existant, à l'échelle des associations comme à celle des acteurs, entre Turcs de Thrace occidentale et de Turquie, il est probable que nombre d'entre eux fréquentent les mêmes lieux de culte qui, en Allemagne, sont principalement gérés par l'association Millî Görüss (Vision nationale) ${ }^{72}$. Nous ne saurions toutefois être catégorique sur ce point, et ce champ d'étude reste lui aussi à explorer.

\section{Annexe : Sadık Ahmet et le contexte politique de l'après crise de Chypre en Thrace}

Sadık Ahmet est né en 1947 dans le village d'Agra, à $30 \mathrm{~km}$ environ à l'est de Komotini. Il obtint en 1967 le diplôme du lycée Celal Bayar de Komotini (lycée de la minorité où l'enseignement est bilingue). Comme cela se faisait couramment à l'époque parmi les étudiants de la minorité, c'est en Turquie qu'il passa le concours d'entrée à l'université, plus facile pour les étrangers. À l'issue d'une première année à la faculté de médecine de l'Université d'Ankara, il fit transférer son inscription à l'Université de Thessalonique sans avoir besoin d'en passer le concours d'entrée ${ }^{73}$. En 1974, ayant obtenu son diplôme, il effectua deux années de service militaire, et une année de service obligatoire en tant que médecin de campagne dans le centre de la Grèce. Puis, dans le cadre d'une spécialisation en chirurgie, il pratiqua deux ans à l'hôpital de Komotini et deux autres années à l'hôpital de la faculté de médecine d'Alexandroupolis (Dedeağaç), jusqu'en 1984. Sadık Ahmet est entré en politique après un échec professionnel : il se vit refuser un poste vacant à l'hôpital de Thessalonique, alors qu'il était le seul candidat.

Il se replia alors sur les structures associatives de la minorité et devint le secrétaire de l'Association des diplômés de Thrace occidentale (Batı Trakya Azmlık Tahsilliler Derneği), à Komotini. À la même époque, les dirigeants de l'association des Turcs de Thrace occidentale de Düsseldorf eurent l'idée de lancer une campagne de signatures pour dénoncer le traitement infligé aux membres de la minorité, particulièrement après la crise de Chypre (1974). Ils désiraient que l'opération soit menée depuis la Thrace. Les personnalités politiques locales approuvèrent le projet, mais leurs divisions empêchèrent la rédaction du texte de la pétition. Au cours de l'année 1986, Sadık Ahmet prit l'initiative de collecter des signatures à la faveur de ses tournées dans les villages, où il pratiquait les circoncisions : il en obtint un millier environ. Notons qu'en sillonant la campagne dans le cadre de son activité professionnelle, Sadık Ahmet a vraisemblablement acquis un solide capital électoral. Le 9 août 1986, il fut interpellé pour excès de vitesse sur l'autoroute

${ }^{72}$ Cette association est liée à la mouvance islamiste de Turquie. Voir sur ce point Amiraux (Valérie), Acteurs de l'islam entre Allemagne et Turquie. Parcours militants et expériences religieuses, Paris : L'Harmattan, 2001.

${ }^{73}$ Le concours d'entrée à l'université en Grèce est particulièrement difficile à réussir, même pour les ètudiants dont le grec est la langue maternelle. Les musulmans de Thrace ont à cet égard un handicap notoire, c'est pourquoi ils bénéficient depuis 1995 d'un quota dans chaque université. 
d'Alexandroupoliset l'arrêtèrent. Il fit ainsi un premier séjour en prison.

Sadık Ahmet s'était rendu une première fois en Allemagne cette année-là, alors qu'il avait déjà commencé à collecter des signatures. Il a rencontré les dirigeants de l'association de Düsseldorf et a laissé le souvenir d'un collaborateur très difficile à gérer, dont il fallait réfréner les excès de langage lors de la rédaction du texte de la fameuse pétition. Le texte en question ne fut pas approuvé par l'Association des diplômés, dont il était le secrétaire. Sadık Ahmet démissionna de son poste après son arrestation, voyant que le comité de direction de l'Association ne le soutenait pas. On voit qu'il était alors une personnalité contestée, aussi bien en Allemagne qu'en Thrace. Sadık Ahmet continua de se rendre en Allemagne après avoir été élu député, pour accompagner les délégations qui se rendaient au siège du Conseil de l'Europe ou organiser des réunions.

Après avoir en vain brigué l'investiture de la Nouvelle Démocratie, Sadık Ahmet se présenta en tant que candidat indépendant aux élections législatives de 1989. Son exemple fut suivi dans le département voisin de Xanthi ; c'était la première fois que des listes indépendantes étaient constituées par des candidats musulmans. Sadık Ahmet fut élu dans le département du Rhodope, mais ces élections furent annulées en raison de l'impossibilité pour la ND de dégager une majorité suffisante au Parlement.

De nouvelles élections furent convoquées en novembre 1989, auxquelles Sadık Ahmet ne put participer en raison d'un vice de procédure lors du dépôt de sa candidature. Le scénario de juin 1989 se reproduisit, en plaçant l'élu turc indépendant, İsmail Rodoplu, en position d'arbitre : il manquait un siège au PASOK pour avoir la majorité absolue au Parlement. Un troisième scrutin eut lieu en avril 1990. La conséquence de cette instabilité politique, et du choc lié à l'émergence de la minorité turque dans la vie politique nationale, fut l'adoption d'une loi instaurant un seuil électoral de $3 \%$ des voix à l'échelle nationale pour qu'un parti puisse être représenté au Parlement.

En janvier 1990, Sadık Ahmet et İbrahim Șerif (actuel mufti dissident de Komotini) furent arrêtés et jugés pour avoir utilisé le mot "Turc" dans leurs tracts et articles publiés au cours de la campagne électorale de novembre 1989. Ce verdict a donné lieu à la manifestation du 29 janvier 1990, qui fut contrée par celle d'une organisation grecque jusqu'alors inconnue, les “Pacifistes de la Thrace”. D'après Vemund Aarbakke, ceux que les grands média grecs ont qualifié de " milliers de musulmans provocateurs " étaient environ 300 et se sont dispersés rapidement lorsque la situation a commencé à s'envenimer. La dégradation et le pillage des habitations et commerces musulmans a duré plusieurs heures, alors que les unités spéciales de la police se contentaient d'observer, voire d'encourager les destructions. La tension fut telle que le consul turc de Komotini et le consul grec d'Istanbul furent chacun reconduits à la frontière les jours suivants.

Sadık Ahmet créa son propre parti en 1991, le Parti de l'amitié, de l'égalité et de la paix (Dostluk, Eșitlik ve Barıș Partisi-DEB), qui n'a qu'une existence symbolique en 
raison du barrage èlectoral introduit en 1990. Lors des différents scrutins auxquels il se présenta, Sadık Ahmet obtint un score jamais égalé par aucun candidat musulman. Il fut également celui qui a officialisé les liens avec le "monde turc", c'està-dire les représentants de la droite nationaliste et radicale turque, dont le discours est en partie fondé sur ceux qu'on appelle les "Turcs de l'extérieur" (diş Türkler), dont le cercle le plus proche englobe les Turcs de Thrace occidentale et ceux de Chypre 74 . Sadık Ahmet est décédé dans un accident de voiture le 25 juillet 1995.

74 Après le coup d'État du 12 septembre 1980, et particulièrement dans les années 199o, la rhètorique de la " mosaïque " turque à l'intérieur et à l'extérieur des frontières est devenue le socle commun du discours politique des partis de gouvernement. 